\title{
Surf-zone vortices over stepped topography
}

\author{
By E. R. JOHNSON AND N. ROBB MCDONALD \\ Department of Mathematics, University College London, Gower Street, \\ London WC1E 6BT, UK
}

(Received 29 July 2003 and in revised form 22 March 2004)

The problem of vortical motions in the surf zone is simplified by taking the bottom topography to be piecewise flat while allowing finite-height jumps in depth between flat regions. The motion of an arbitrary number of singular vortices is cast into Hamiltonian form and the rule for relating Hamiltonians in conformally equivalent domains derived. Examples are given of a singular vortex pair colliding head-on with a step, of a vortex propagating along a curved coast to cross a step, and of a vortex being swept past a circular island straddling a step. Surf-zone vortices are then modelled as finite-area vortex patches and their motion followed by contour dynamics. It is shown that the paths of singular vortices can yield highly accurate explicit predictions of the paths of the centroids of vortex patches. Possible applications to surf-zone rip currents are noted.

\section{Introduction}

Recently several studies have highlighted the importance of vortical motions in the surf zone. Their presence is perhaps not surprising given that surf-zone hydrodynamics is almost two-dimensional and that the cascade of energy to large scales in two-dimensional fluid motion is often characterized by persistent and stable eddy structures. Peregrine (1998) presents evidence for vortex structures arising from along-shore currents and argues that, in particular, rip currents arise from the pairing of opposite signed vortices in the form of a propagating dipole. Further, Peregrine $(1998,1999)$ derives formulae for the generation of the vorticity required to form such structures by bores and breaking waves. Numerical simulation of the fully nonlinear equations appropriate to the surf zone has also demonstrated the ubiquity of vortices. For example, Özkan-Haller \& Kirby (1999) numerically solve the shallow-water equations (with additional terms to account for forcing and friction) in a plane beach geometry and find that, for typical parameter values, vortices evolve with length scales of $O(100 \mathrm{~m})$ and time scales of $O(100 \mathrm{~s})$. In their experiments, oppositely signed vortices are frequently observed to pair up and propagate offshore as dipoles. Numerical experiments showing evidence for robust vortical motions in a surfzone environment have also been performed by other researchers, for example, Slinn et al. (1998) and Chen et al. (1999). In a theoretical and numerical study, Bühler \& Jacobson (2001) consider the generation of dipolar vortex structures by breaking waves on a barred beach.

The presence of quasi-two-dimensional vortices in the surf zone has lead several authors to investigate their properties theoretically. It was noted in passing by Johnson (1978), and discussed in greater detail by Peregrine (1998), that a vortex on a uniformly sloping beach (i.e. a 'wedge'-shaped domain) is topologically equivalent to part of a vortex ring and therefore has the ability to self-propagate parallel to the shore owing 
to its image. Thorpe \& Centurioni (2000) also discuss the relation of vortices in a wedge and vortex rings, and their application to surf-zone vortices. Richardson (2000) presents an asymptotic study of surf-zone vortices over varying bottom topography in the limit that the vortices are small compared to the length scale of the topographic variations. Richardson (2000) finds that to leading order an isolated vortex propagates along isobaths at speed given by the local gradient in the logarithm of the depth. Bühler \& Jacobson (2001) use the conservation of potential vorticity to construct a simple analytic model for calculating the propagation velocity of surf-zone vortices (occurring in dipolar pairs) taking into account the mutual self-advection mechanism and the effects of the sloping beach.

In the present work an analytical and numerical model is developed for surfzone vortices moving due the presence of other vortices, variable topography and background irrotational flow. Following previous works on surf-zone vortices by Richardson (2000) and Thorpe \& Centurioni (2000) the fluid motion is assumed to be governed by the shallow-water equations with small Froude number so that the free surface is flat (i.e. a rigid lid). However, a different depth profile is considered here, namely piecewise flat topography. Unlike the quasi-geostrophic equations of motion frequently used in geophysical fluid dynamics (Pedlosky 1986), the step changes in depth between flat regions can be comparable to the average depth of the fluid. This choice allows explicit Green's functions for singular vortices to be derived simply for many geometries. It is also motivated by the observation that many, if not most, beaches are barred and so could be represented in a first approximation by an array of infinitely long steps. Near such steep topography vertical fluid velocities may become comparable to horizontal velocities, violating the shallow-water approximation. However, provided the horizontal extent of the vortex is much larger than the water depth, the effect of depth variations on the vortex as a whole is still modelled well by the shallow-water equations (see, for example, Johnson 1990, 1993 for a comparison of flows over finite-amplitude stepped and smooth topography).

The problem discussed in detail here is that of a vortex pair (comprising singular vortices or patches of uniform vorticity) propagating at normal incidence to a infinitely long step. Consideration of vortex pairs is motivated by their frequent occurrence in the numerical simulations of Özkan-Haller \& Kirby (1999) and Chen et al. (1999), and their generation by breaking waves (Bühler \& Jacobson 2001). Moreover, Peregrine (1998) argues that rip currents are closely associated with self-propagating vortex pairs. This is supported by the field observations of Smith \& Largier (1995). Further, it is known that dipolar vortex structures are generally more robust than their monopolar counterparts (Gorshkov, Ostrovsky \& Soustova 2000) and are therefore more likely to be of importance in the dynamics of the surf zone. The ability of dipoles to self-propagate (and so transport fluid) also provides a natural mechanism for the dipole to approach and interact with the topography.

Section 2 gives a Hamiltonian formulation for the motion of an arbitrary number of singular vortices above piecewise flat topography and a rule for relating the Hamiltonians in conformally equivalent domains. Section 3 applies these results to a singular vortex pair colliding head-on with an infinite rectilinear step, to vortex trajectories along a curved coast with an alongshore depth change and to trajectories, in the presence of a background flow, past a circular island straddling a step. The Green's function for a singular vortex is obtained by requiring continuity of the pressure at the step and the volume flux normal to the step. It is shown that depending on whether the vortex lies on the deep or the shallow side, the step acts as 
either a partial 'wall' or 'anti-wall'. That is the vortex sees either an image (which has the opposite sign to the vortex) or an 'anti-image' (which has the same sign as the vortex). The strength of the image in either case depends on the height of the step and in the limit where the step height approaches the depth of the fluid the image becomes that of a conventional image in a wall. In addition to allowing the trajectories of singular vortices to be calculated, the relatively simple form of the Green's function allows contour surgery (Dritschel 1988) to be used to study the motion of patches of constant vorticity. Vortex patches are more realistic models for surf-zone vortices since, unlike singular vortices, they can deform, split and merge. One of the principal aims of this study is to compare the behaviour of singular vortices and vortex patches and $\S 4$ presents such comparisons showing that for head-on collisions the paths of singular vortices from $\S 3$ generate highly accurate explicit predictions of the paths of vortex patch centroids. Section 5 discusses briefly the results and their applications.

\section{Equations and Hamiltonian structure}

Mass conservation in the low Froude number (rigid lid) limit of the shallow-water equations allows the introduction of a volume flux streamfunction $\psi$ so that the horizontal components of velocity are given by

$$
(u, v)=H^{-1}\left(\psi_{y},-\psi_{x}\right),
$$

where $H(x, y)$ is the local fluid depth, which is independent of time in this limit. The motion is then governed by the conservation of potential vorticity which can be written as

$$
q_{t}+\boldsymbol{u} \cdot \nabla q=0,
$$

where $q=\zeta / H$ is the potential vorticity for $\zeta=v_{x}-u_{y}$, the vertical component of vorticity. The instantaneous distribution of $q$ determines the velocity field through (2.1) and the vorticity-streamfunction relation

$$
-\nabla \cdot\left(H^{-1} \nabla \psi\right)=\zeta
$$

Written in this form the operator in (2.3) is self-adjoint and so the associated Green's function $G\left(z, z_{0}\right)$ (determined by solving (2.3) subject to zero boundary conditions on solid walls and at large distances, with right-hand side a point singularity - the product of delta functions $\delta\left(x-x_{0}\right) \delta\left(y-y_{0}\right)$ where $z=x+\mathrm{i} y$ and $\left.z_{0}=x_{0}+\mathrm{i} y_{0}\right)$ is symmetric in the sense that $G\left(z, z_{0}\right)=G\left(z_{0}, z\right)$. Denote the symmetric linear operator determined by $G$ by $\mathscr{G}$ so $\psi=\mathscr{G} \zeta$. Then the kinetic energy of the motion can be written

$$
E=\frac{1}{2} \int_{\mathscr{D}}\left(u^{2}+v^{2}\right) H \mathrm{~d} x \mathrm{~d} y=\frac{1}{2} \int \zeta \mathscr{G} \zeta \mathrm{d} x \mathrm{~d} y=\frac{1}{2} \int H q^{\mathscr{G}}(H q) \mathrm{d} x \mathrm{~d} y,
$$

where the first integral is over the flow domain $\mathscr{D}$ but the further integrals are over the support of $\zeta$, taken to be finite here, and so are absolutely convergent. Equation (2.2) shows that $E$ is a constant of the motion. For general $H, E$ is the only constant of the motion.

It is possible to make further progress on the general motion of distributed vortices above arbitrary topography by following Johnson (1978) where (2.1) and (2.2) are interpreted as requiring that the potential vorticity evolves in a stretched frame so as to remain a rearrangement of some initial distribution. In particular it is noted there that if the depth varies linearly with an offshore coordinate then the problem reduces 
precisely to that of an axisymmetric vortex ring and so all the classical results for vortex rings carry over directly. This observation has been discussed in greater detail by Peregrine (1998) and Thorpe \& Centurioni (2000). The motion of finite-area vortex patches is considered here in $\S 4$. However, as for vortex rings, much information follows from considering thin vortices. This is more involved than the modelling of constant-depth vortex patches by singular vortices as in Johnson \& McDonald $(2003 a, b)$ since the propagation speed of a vortex becomes logarithmically infinite as its radius decreases if the depth is not locally constant (Kelvin, in an appendix to Tait 1887 for the linear beach; Richardson 2000 for general beaches). These computational, analytical and expositional difficulties are avoided below by restricting attention to topographies that are piecewise flat and following Saffman's (1992) discussion for two-dimensional (constant-depth) motion. The extension to continuous distributions and topography will be given elsewhere.

Consider a system of $N$ singular vortices of potential vorticities $Q_{1}, \ldots, Q_{N}$ and instantaneous positions $z_{1}=x_{1}+\mathrm{i} y_{1}, \ldots, z_{N}=x_{N}+\mathrm{i} y_{N}$, none of which lies initially above a discontinuity of $H$. The associated vorticity distribution can thus be written

$$
\zeta\left(z ; z_{1}, \ldots, z_{N}\right)=\sum_{k=1}^{N} H\left(z_{k}\right) Q_{k} \hat{\delta}\left(x-x_{k}\right) \hat{\delta}\left(y-y_{k}\right),
$$

where $\hat{\delta}$ denotes the Dirac delta function. Take $H$ to be piecewise constant and let the streamfunction of any irrotational background flow be $\psi_{b}(z)$. The streamfunction for the whole flow follows from (2.3) by summing the contributions of the individual vortices as

$$
\psi\left(z ; z_{1}, \ldots, z_{N}\right)=\psi_{b}(z)+\sum_{k=1}^{N} H\left(z_{k}\right) Q_{k} G\left(z, z_{k}\right) .
$$

Above flat regions $G$ is harmonic. Requiring the pressure and mass flux to be continuous across discontinuities in $H$ gives continuity conditions on $G$ and $(1 / H) \partial G / \partial n$ (where $\partial / \partial n$ is the normal derivative). The Green's function for a finiteheight circular cylinder is given in Johnson (1978) and for a finite-height rectilinear step below. Integrating (2.3) over a vanishingly small disk centred on $z_{k}$ shows that to leading order

$$
G\left(z, z_{k}\right) \rightarrow-\left(H\left(z_{k}\right) / 2 \pi\right) \log \left|z-z_{k}\right| \quad \text { as } \quad z \rightarrow z_{k},
$$

and so the function $g\left(z, z_{k}\right)$ defined by

$$
g\left(z, z_{k}\right)=G\left(z, z_{k}\right)+\left(H\left(z_{k}\right) / 2 \pi\right) \log \left|z-z_{k}\right|,
$$

is differentiable everywhere $H$ is. The regular function $g$ gives the streamfunction at $z$, due to the vortex at $z_{k}$, needed to satisfy the boundary conditions on the flow and the differential equation away from the vortex. It can be regarded as the image of vortex $k$ in the topography and boundaries. Form (2.4) suggests introducing the Hamiltonian

$$
\begin{aligned}
\mathscr{H}\left(z_{1}, \ldots, z_{N}\right)=\sum_{j=1}^{N} Q_{j} H\left(z_{j}\right) \psi_{b}\left(z_{j}\right)+\sum_{j=1}^{N} \sum_{k=j+1}^{N} Q_{j} H\left(z_{j}\right) Q_{k} H\left(z_{k}\right) G\left(z_{j}, z_{k}\right) \\
+\frac{1}{2} \sum_{j=1}^{N} Q_{j}^{2} H^{2}\left(z_{j}\right) g\left(z_{j}, z_{j}\right) .
\end{aligned}
$$


Differentiating (2.9), noting that $(\mathrm{d} / \mathrm{d} t) H\left(z_{j}\right)=0$ and $G$ is symmetric, gives

$$
\frac{\mathrm{d}}{\mathrm{d} t} \mathscr{H}\left(z_{1}, \ldots, z_{N}\right)=\sum_{j=1}^{N} Q_{j} H\left(z_{j}\right)\left(\frac{\partial \psi_{j}}{\partial x_{j}} \frac{\mathrm{d} x_{j}}{\mathrm{~d} t}+\frac{\partial \psi_{j}}{\partial y_{j}} \frac{\mathrm{d} y_{j}}{\mathrm{~d} t}\right),
$$

where

$$
\psi_{j}\left(z_{1}, \ldots, z_{N}\right)=\psi_{b}\left(z_{j}\right)+Q_{j} H\left(z_{j}\right) g\left(z_{j}, z_{j}\right)+\sum_{k=1}^{N} Q_{k} H\left(z_{k}\right) G\left(z_{j}, z_{k}\right),
$$

and the prime on the sum means that the term $k=j$ is omitted. It follows immediately that the velocity components derived from (2.6) approach those of (2.11) at vortex positions but with the singular self-advection term (2.7) absent from $\psi_{j}$ as required. The function $\psi_{j}$ is a thus a streamfunction for the velocity of propagation of the $j$ th vortex, with

$$
\frac{\mathrm{d} x_{j}}{\mathrm{~d} t}=H^{-1}\left(z_{j}\right) \frac{\partial \psi_{j}}{\partial y_{j}}, \quad \frac{\mathrm{d} y_{j}}{\mathrm{~d} t}=-H^{-1}\left(z_{j}\right) \frac{\partial \psi_{j}}{\partial x_{j}},
$$

and (2.10) shows that $\mathscr{H}$ is indeed a constant of the motion. The evolution of the vortex field follows directly by integrating the path equations (2.12).

If only one vortex is present $(N=1)$ then the double sum in (2.9) is absent and the vortex is constrained to travel along the level curves of $\mathscr{H}$, i.e. along the lines $\psi_{b}\left(z_{1}\right)+Q_{1} H\left(z_{1}\right) g\left(z_{1}, z_{1}\right)=$ constant. When two vortices are present then their paths are in general more complex. However, if the depth profile is axisymmetric then the angular impulse is conserved or if the depth is independent of $y$ the impulse in the $x$-direction is conserved (Johnson 1978). This constrains the motion further and in the example of $\S 3.1$ allows the paths to be determined completely for a vortex pair travelling perpendicularly to rectilinear topography.

\subsection{Conformal invariance and a Routh rule}

Let $\hat{z}=f(z)$ define a conformal map from the flow domain $\mathscr{D}$ to the corresponding domain $\widehat{\mathscr{D}}$ of the complex- $\hat{z}$-plane. Equation (2.3) is not in general invariant under conformal mappings. However, direct evaluation (for example using the scale factors for the transformation as in Johnson 1987) shows that (2.3) is invariant for general $H(x, y)$ when the vorticity $\zeta$ is a sum of delta functions as in (2.5). In particular $\widehat{G}\left(\hat{z}, \hat{z}_{0}\right)=G\left(z, z_{0}\right)$, with $\widehat{G}$ vanishing on the solid boundaries of $\widehat{\mathscr{D}}$ as required since $G$ vanishes at the corresponding points on $\mathscr{D}$. The instantaneous streamfunction, and thus flow field, due to singular potential vortices of strengths $Q_{1}, \ldots, Q_{N}$ at points $z_{1}, \ldots, z_{N}$ maps to the flow field due to vortices of exactly the same strengths at the points $\hat{z}_{1}=f\left(z_{1}\right), \ldots, \hat{z}_{N}=f\left(z_{N}\right)$. This result is the extension to general, smooth or stepped, finite-amplitude topography of the classical result for two-dimensional ideal fluid flow, to which it reduces if the depth is constant. It remains valid here even in the absence of a complex velocity potential.

The advection equation (2.2) is not invariant under conformal transformations $\dagger$ and thus, although the instantaneous vortex-induced flow fields in $\mathscr{D}$ and $\widehat{\mathscr{D}}$ correspond, the trajectories of the vortices themselves differ. It is again convenient to restrict attention to piecewise flat topography with no vortex lying above a discontinuity

$\dagger$ A linearized combined form of (2.2) and (2.3) for wave motion over finite-height topography in a rotating frame is however invariant (Johnson 1985, 1987). 
of $H$. Then the topography is flat within a sufficiently small neighbourhood $\mathscr{N}_{k}$, say, with image $\widehat{\mathscr{N}}_{k}$, surrounding vortex $k$. Since $H$ is constant within $\mathscr{N}_{k}$ and $\widehat{\mathscr{N}}_{k}$, complex velocity potentials can be introduced for the motion there. The invariance of the vortex-induced streamfunction means that these correspond except at the vortex where they are singular, of form (2.7). Introduce $w_{k}$ as the complex velocity potential in $\mathscr{N}_{k}$ minus the local vortex term and $\hat{w}_{k}$ as the complex potential in $\widehat{\mathscr{N}}_{k}$ minus the local vortex term. Then

$$
-\mathrm{i}\left(Q_{k} / 2 \pi\right) H^{2}\left(z_{k}\right) \log \left(z-z_{k}\right)+w_{k}(z)=-\mathrm{i}\left(Q_{k} / 2 \pi\right) \hat{H}^{2}\left(\hat{z}_{k}\right) \log \left(\hat{z}-\hat{z}_{k}\right)+\hat{w}_{k}(\hat{z}),
$$

with $w_{k}$ and $\hat{w}_{k}$ analytic in $\mathscr{N}_{k}$ and $\widehat{\mathscr{N}}_{k}$. Expanding about $\hat{z}$ and $\hat{z}_{k}$ gives

$$
w_{k}(z)=\hat{w}_{k}(\hat{z})-\mathrm{i}\left(Q_{k} / 2 \pi\right) H^{2}\left(z_{k}\right)\left\{\log \left[f^{\prime}\left(z_{k}\right)\right]+\frac{1}{2}\left(z-z_{k}\right) f^{\prime \prime}\left(z_{k}\right) / f^{\prime}\left(z_{k}\right)+\cdots\right\} .
$$

Differentiating with respect to $z$ and taking the limit $z \rightarrow z_{k}$ relates the velocities of vortex $k$ in the two domains through

$$
u_{k}-\mathrm{i} v_{k}=f^{\prime}\left(z_{k}\right)\left(\hat{u}_{k}-\mathrm{i} \hat{v}_{k}\right)-\mathrm{i}\left(Q_{k} / 4 \pi\right) H\left(z_{k}\right) f^{\prime \prime}\left(z_{k}\right) / f^{\prime}\left(z_{k}\right),
$$

which reduces in constant-depth flow to the Routh rule (Routh 1881; Saffman 1992) for vortex motion. As expected, this differs from the relation for the non-singular part of the instantaneous fluid velocity, which omits the final term on the right. With velocity components derived in a simple domain the equations for vortex trajectories in a more complicated domain can be integrated numerically to give the vortex paths there.

The complex velocities in (2.15) are not in general analytic functions of $z_{k}$ and so no complex potential for the vortex motion, as opposed to the induced instantaneous flow field, exists. However, following Saffman (1992) for constant-depth flow,

$$
\begin{aligned}
\mathrm{d} \psi_{k} & =\frac{\partial \psi_{k}}{\partial x_{k}} \mathrm{~d} x_{k}+\frac{\partial \psi_{k}}{\partial y_{k}} \mathrm{~d} y_{k}=\operatorname{Im}\left\{H\left(z_{k}\right)\left(u_{k}-\mathrm{i} v_{k}\right) \mathrm{d} z_{k}\right\} \\
& =\operatorname{Im}\left\{H\left(\hat{z}_{k}\right)\left(\hat{u}_{k}-\mathrm{i} \hat{v}_{k}\right) \mathrm{d} \hat{z}_{k}-\mathrm{i}\left(Q_{k} / 4 \pi\right) H^{2}\left(z_{k}\right)\left[f^{\prime \prime}\left(z_{k}\right) / f^{\prime}\left(z_{k}\right)\right] \mathrm{d} z_{k}\right\} \\
& =\mathrm{d} \widehat{\psi}_{k}-\left(Q_{k} / 4 \pi\right) H^{2}\left(z_{k}\right) \mathrm{d}\left[\log \left|f^{\prime}\left(z_{k}\right)\right|\right],
\end{aligned}
$$

using (2.15) and $\mathrm{d} \hat{z}_{k}=f^{\prime}\left(z_{k}\right) \mathrm{d} z_{k}$, where Im denotes the imaginary part and $\widehat{\psi}_{k}$ is the streamfunction for the velocity of vortex $k$ in domain $\widehat{\mathscr{D}}$. Thus

$$
\widehat{\psi}_{k}\left(\hat{z}_{1}, \ldots, \hat{z}_{N}\right)=\psi_{k}\left(z_{1}, \ldots, z_{N}\right)+\left(Q_{k} / 4 \pi\right) H^{2}\left(z_{k}\right) \log \left|f^{\prime}\left(z_{k}\right)\right|,
$$

and the Hamiltonians in the two domains are related through

$$
\widehat{\mathscr{H}}\left(\hat{z}_{1}, \ldots, \hat{z}_{N}\right)=\mathscr{H}\left(z_{1}, \ldots, z_{N}\right)+\frac{1}{4 \pi} \sum_{k=1}^{N} Q_{k}^{2} H^{3}\left(z_{k}\right) \log \left|f^{\prime}\left(z_{k}\right)\right|,
$$

with the Hamiltonian $\widehat{\mathscr{H}}$ a constant of the motion in $\widehat{\mathscr{D}}$.

\section{A rectilinear step}

For the finite step change in depth, where $H(x, y)=H^{+}$in $y>0$ and $H^{-}$in $y<0$, the Green's function has the succinct expression in complex form,

$$
G\left(z, z_{0}\right)= \begin{cases}-(1 / 2 \pi) H\left(z_{0}\right)\left[\log \left|z-z_{0}\right|+\alpha \log \left|z-\bar{z}_{0}\right| \operatorname{sgn} y\right], & y y_{0}>0, \\ -(1 / 2 \pi) \beta \log \left|z-z_{0}\right|, & y y_{0}<0\end{cases}
$$


where $\bar{z}$ denotes the complex conjugate of $z$, and

$$
\alpha=\frac{H^{-}-H^{+}}{H^{-}+H^{+}}=\frac{\gamma-1}{\gamma+1}, \quad \beta=\frac{2 H^{-} H^{+}}{H^{-}+H^{+}}=\frac{2 \gamma H^{+}}{\gamma+1},
$$

for depth ratio $\gamma=H^{-} / H^{+}$. Similar forms are given in Bidlot \& Stern (1994) and Richardson (2000). Note that $G$ is symmetric as expected and $g$ derived through (2.8) is non-singular. When the depths are the same the escarpment vanishes $(\alpha, \beta)=(0, H)$ and the solution becomes that of a single isolated vortex. When the fluid depth vanishes in $y<0$, so $H^{-}=0$ and the escarpment becomes a vertical wall throughout the fluid depth, $(\alpha, \beta)=(-1,0)$ : there is no motion in $y<0$ and that in $y>0$ is given by the usual image in the wall $y=0$. For reducing depth $(\gamma<1)$ form (3.1) can be interpreted in $y>0$ as a weakened image (in the step) of strength $\alpha$ and in $y<0$ as a partially shielded vortex of reduced strength $\beta$. For increasing depth $\gamma>1$ the downward escarpment acts as a partial 'anti-wall' so the image term in $y>0$ is of the same sign as the original vortex and the shielded vortex in $y<0$ is stronger than the original vortex. The relative strength, $\alpha$, of the image lies between -1 for a wall and +1 for an infinitely deep downward step (and so $0<\beta<2 H^{+}$). Here, as in the classical case of vortices near a wall, the images exist to give the correct alteration (relative to the flow in the absence of the step) in flux across the step: a negative image decreases the flux at an upward step and a positive image increases it at a downward step.

\subsection{A singular vortex pair with step topography}

Consider the above topography with a singular vortex of constant potential vorticity $Q$ lying at $\left(x_{0}, y_{0}\right)$ and suppose there is an impermeable coast aligned along $x=0$. This is equivalent to taking the vortex as forming half of a pair with its accompanying equal and opposite vortex instantaneously at $\left(-x_{0}, y_{0}\right)$. Symmetry about the line $x=0$ means that it is sufficient to consider the motion of either vortex. The streamfunction for the motion is given by substituting (3.1) into (2.6) and the time evolution of the flow can be followed by integrating the equations (2.12). If the trajectories themselves, as opposed to the temporal development of the flow, are alone of interest, then substituting (3.1) into the Hamiltonian (2.9) gives, dropping the now redundant subscript,

$$
\begin{aligned}
\mathscr{H}(z) & =\frac{1}{2} Q^{2} H^{2}(z) g(z, z)+\frac{1}{2}(-Q)^{2} H^{2}(-\bar{z}) g(-\bar{z},-\bar{z})+Q(-Q) H(z) H(-\bar{z}) G(z,-\bar{z}) \\
& =\left(Q^{2} H^{3}(z) / 2 \pi\right)[-\alpha \log |2 y| \operatorname{sgn} y+\log |2 x|+\alpha \log |2 z| \operatorname{sgn} y] .
\end{aligned}
$$

The curves of constant $\mathscr{H}$, along which the vortex is constrained to move, are thus

$$
x=A|y / r|^{\alpha \operatorname{sgn} y},
$$

for $r=\left(x^{2}+y^{2}\right)^{1 / 2}$, the distance of the vortex from the origin, and positive constants A. Figure 1(a) shows some of these trajectories for a positive vortex moving in the $-y$-direction. The simplicity of this geometry means that all trajectories can be scaled so that the vortices at large $y$ are at unit distance from the wall (by scaling distances on $A$ in (3.4)). If the step is absent (so $H^{+}=H^{-}$and $\alpha=0$ ) then the paths are straight lines parallel to the $y$-axis, as expected. If $H^{-}=0$ so that the step becomes a wall (and $\alpha=-1$ ) then the well-known (Lamb 1932) paths are the curves $x^{-2}+y^{-2}=$ constant. To find the shape of the paths for general $\alpha$ (with $|\alpha| \leqslant 1$ ) note that for large $y$ at fixed $x, r$ can be identified with $y$ in (3.4) and so $x \rightarrow A$, as $y \rightarrow \infty$. Now suppose that $x$ approaches zero more slowly than $y$ as $y$ decreases to zero, i.e. the vortex approaches the step. This will certainly be so if the vortex were to cross the step away 

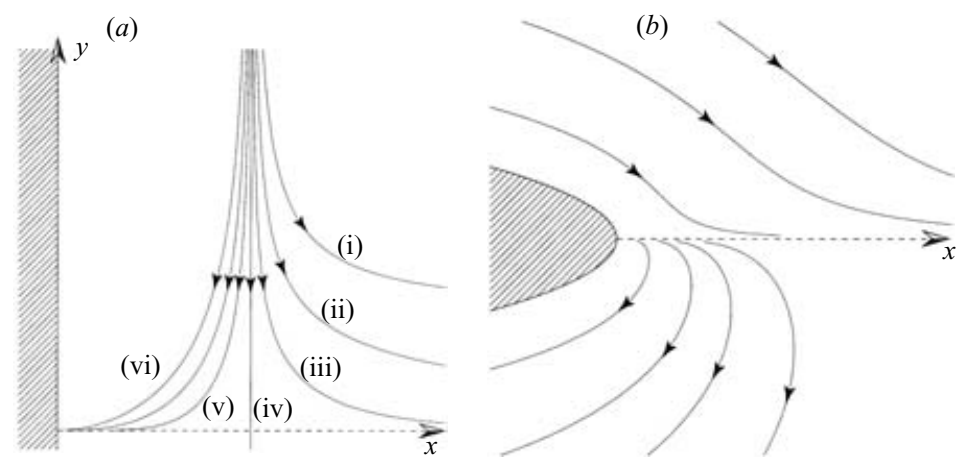

FIgURE 1. (a) Trajectories for a positive singular vortex moving in the $-y$-direction. An impermeable coast lies along $x=0$ or, equivalently, the vortex is the right half of a singular vortex pair placed symmetrically about $x=0$. The step change in depth lies along $y=0$ and is shown dashed. Each trajectory is scaled so that the vortex starts a unit distance from the wall when $y \gg 1$. Trajectories are shown for depth ratio $\gamma=H^{-} / H^{+}$equal to: (i) 0 , a solid wall along $y=0$; (ii) 0.3 ; (iii) 0.6 ; (iv) 1 , when the step is absent and the vortex propagates parallel to the wall; (v) $1.5 ; 3$; and (vi) 100 , when the flow is effectively infinitely deep after the step. Trajectories for downward steps $(\gamma>1)$ end at the origin. Trajectories for upward steps $(\gamma<1)$ asymptote to the step for $x \gg 1$. (b) Trajectories for a parabolic coast. Once the coast shape is fixed here the initial vortex position cannot be scaled to unit displacement from the wall for different $\gamma$ and so becomes an additional parameter in the solution. Here $\gamma=0.5$ and trajectories in both $y>0$ and $y<0$ are shown.

from the origin as then $y$ would vanish for some non-zero $x$. Now $r$ can be identified with $x$ in (3.4) and so

$$
x \rightarrow A^{1 /(1+\alpha)} y^{\alpha /(1+\alpha)} \quad \text { as } \quad y \rightarrow 0 .
$$

Thus if $\alpha>0, x$ approaches zero more slowly than $y$ and (3.5) gives the vortex path near a downward step. The smaller the step the closer the vortex approaches before turning towards the origin. It does not cross the step at any non-zero $x$. If $\alpha<0, x$ increases as $y \rightarrow 0$ and (3.5) gives the vortex path near an upward step. Again, the smaller the step the closer the vortex approaches before turning to move outwards parallel to the step and the more rapidly the vortex asymptotes towards the step, and again, the vortex does not cross the step at any finite $x$. No matter how weak the image in the step, eventually the vortex approaches sufficiently closely for the image to dominate the vortex motion. The singular vortex model precludes vortices from crossing finite steps. It is shown in $\S 4$ that the less idealized model of finite-area vortex patches does allow vortices to cross steps.

Once paths in this simple domain are known those in more complicated domains can be constructed using (2.18). Consider the conformal mapping of the half-plane $\operatorname{Re} z>0$ defined by

$$
\hat{z}=(z+1)^{2}, \quad z=\hat{z}^{1 / 2}-1, \quad \mathrm{~d} \hat{z} / \mathrm{d} z=2(z+1) .
$$

This maps the linear coast in the $z$-plane to a parabolic coast in the $\hat{z}$-plane leaving the step unchanged along $y=\hat{y}=0$. The lack of left-right symmetry in the $\hat{z}$-plane means that the interpretation of the vortex as half of a pair is lost: $\mathscr{H}(z)$ must be calculated as the Hamiltonian for a single vortex off a straight coast and so its value 
(a)

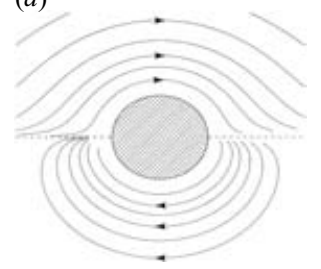

(b)

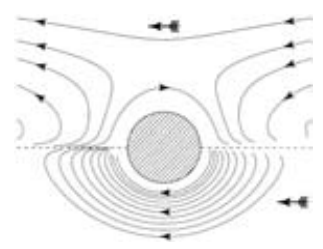

(c)

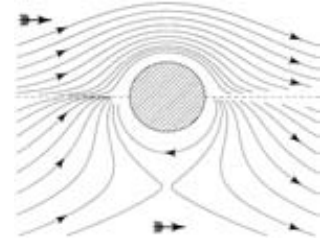

Figure 2. Paths for singular vortices near a circular island. The depth halves $(\gamma=0.5)$ in moving from $y>0$ to $y<0$ across the step $y=0$ (dashed). ( $a$ ) No background flow. Positively signed vortices move clockwise around the island, smoothly passing the island in $y>0$ and in $y<0$ emerging from $\left(1,0^{-}\right)$to pass to $\left(-1,0^{-}\right)$. $(b)$ With background flow from right to left (for positively signed vortices), indicated here, and in (c), by the bold complete arrows in each half-plane. Trajectories in $y<0$ are relatively unaffected. A saddle point appears in $y>0$ with the associated separatrix dividing vortices that are swept past the island from those influenced by the step and island. (c) With background flow from left to right. Trajectories in $y>0$ are relatively unaffected. The separatrix associated with the saddle point in $y<0$ again divides vortices that are swept past the island from those influenced by the step and island.

halves. Then (2.18) and (3.3) give

$$
\hat{\mathscr{H}}(\hat{z})=\frac{1}{2} \mathscr{H}(z)+\frac{Q^{2} H^{3}(z)}{4 \pi} \log |z+1|=\left.\frac{Q^{2} H^{3}(z)}{4 \pi} \log || \frac{y}{z}\right|^{-\alpha \operatorname{sgn} y} x(z+1) \mid .
$$

Figure $1(b)$ shows trajectories for this parabolic coast for when the depth halves in moving from $y>0$ into $y<0(\gamma=0.5)$. Here the initial displacement of the vortex from the coast cannot be scaled from the motion. Note that the trajectories are not simply the mapped forms of the original trajectories as they eventually become parallel to the coast, as expected from physical considerations. The transformation

$$
\hat{z}=(1+z) /(1-z), \quad z=(\hat{z}-1) /(\hat{z}+1), \quad \mathrm{d} \hat{z} / \mathrm{d} z=2 /(1-z)^{2},
$$

maps the half-plane $\operatorname{Re} z>0$ to the exterior of the unit circle in the $\hat{z}$-plane with the step lying again along $\hat{y}=0$. If additionally a uniform stream from the right, parallel to the step, is superposed the Hamiltonian becomes

$$
\hat{\mathscr{H}}(\hat{z})=\frac{1}{2} \mathscr{H}(z)-\frac{Q^{2} H^{3}(z)}{2 \pi} \log |1-z|-U H(\hat{z}) \hat{y}\left(1-|\hat{z}|^{-2}\right) .
$$

The final term is the background irrotational shallow-water flow satisfying the same continuity conditions across the step as $G$. Figure 2 gives examples of vortex paths with and without the background flow. As in Johnson \& McDonald $(2003 a, b)$ a background flow opposing the image-induced vortex propagation introduces a saddle point in the Hamiltonian with the associated separatrix dividing vortices whose paths pass sufficiently far from the island and step so as to be relatively unaffected from those dominated by image vorticity. In figure $2(b)$ vortices closer than about two cylinder radii to the step are turned back to return upstream. Similarly, in $(c)$ vortices closer than about three cylinder radii to the step are captured by the singularity at $(-1,0)$. Form (3.9) shows that the width of these interaction regions varies with the ratio of background flow speed to vortex strength.

\section{Contour dynamics for patches of constant potential vorticity}

To follow the motion of distributed vortices it is generally necessary to invert (2.3) numerically and use the derived velocity field in (2.2) to advect the potential 
vorticity. For piecewise-constant distributions of potential vorticity contour dynamics provides an especially efficient and accurate method to do this. The integrations here are based on Dritschel's (1988) contour surgery algorithm which allows contours to split and merge and thus enables integrations to be carried out to much longer times. Modifications are required to allow for the different form of the Green's function on each side of the step. For a vortex that lies entirely on one side of the step an image vortex is constructed in accordance with (3.1) to calculate the effect of the step. A vortex that straddles the step requires two images: one for the part of the vortex in deep water and the other for the part in shallow water. The effect of the image on the velocity field is weighted by $\alpha$, again in accordance with (3.1). If the vortex crosses the step its relative vorticity $\zeta$ adjusts to meet the demand of constant $\zeta / H$. This effect is built into the algorithm and a consequence is that as $H$ changes, the area of the vortex also changes by an amount depending on the depth ratio. This is quite noticeable in the results presented below where, for example, the area of a vortex pair travelling from deep to shallow water increases. As a test of accuracy of the method the conserved quantity of the product of vorticity and area (i.e. circulation) for each vortex is monitored and is found to change by less than $1 \%$ throughout all integrations.

The method is independent of the number and disposition of the vortices. However, for comparison with $\S 3.1$, let the depth profile be precisely the single rectilinear step of $\S 3$ with an initial vorticity distribution consisting of a pair of circular (radius $a$ ) vortices of equal and opposite strengths $\pm Q$, located in $y>0$, symmetrically about $x=0$, with centres separated by $\Delta$ (figure 3 ). The flow remains symmetric about $x=0$ at all succeeding times. It is shown, for constant-depth flow, in Johnson \& McDonald $(2003 a)$ that provided vortices remain circular their centroids are constrained to follow the paths of singular vortices with the same circulation and the argument there extends without modification to piecewise-constant depth profiles for any vortex that is not straddling a discontinuity in $H$. Near the step it is convenient to consider separately the components of velocity perpendicular and parallel to the step. Both for a finite-area vortex and for its equivalent singular vortex the velocity component perpendicular to the step is due to the other vortex that makes up its pair and the component parallel to the step is due to the image of the vortex itself in the step. To leading order in centroid separation the component of velocity in the negative $y$-direction is the same for the finite-area vortex and the singular vortex and is equivalent to a uniform background flow of fixed magnitude (inversely proportional to the centroid separation). The ratio of this background cross-step velocity to the along-step velocity component of a vortex determines the angle at which the vortex approaches or crosses a step. To estimate the along-step component of vortex velocity it is sufficient to ignore its partner and consider the vortex in isolation. A near-circular finite-area vortex not straddling the step moves along it (due to its image in the step) at a speed that varies inversely with the distance of the vortex centroid from the step. Once the vortex straddles the step two images appear as given by (3.1). The part of a vortex on the deep side experiences an oppositely signed partial image on the shallow side and the part of the vortex on the shallow side experiences a same-signed partial image on the deep side. The images reinforce to move a positive vortex so that shallow water lies to its right and a negative vortex so that shallow water lies to its left. Since the distance between the centroids of the vortex parts and their images does not vanish, this along-step velocity is finite. Detailed computations of steadily propagating vortex patches are given in Hinds, Johnson \& McDonald (2004) where it is shown that the along-step velocity for a given size vortex straddling a step increases 

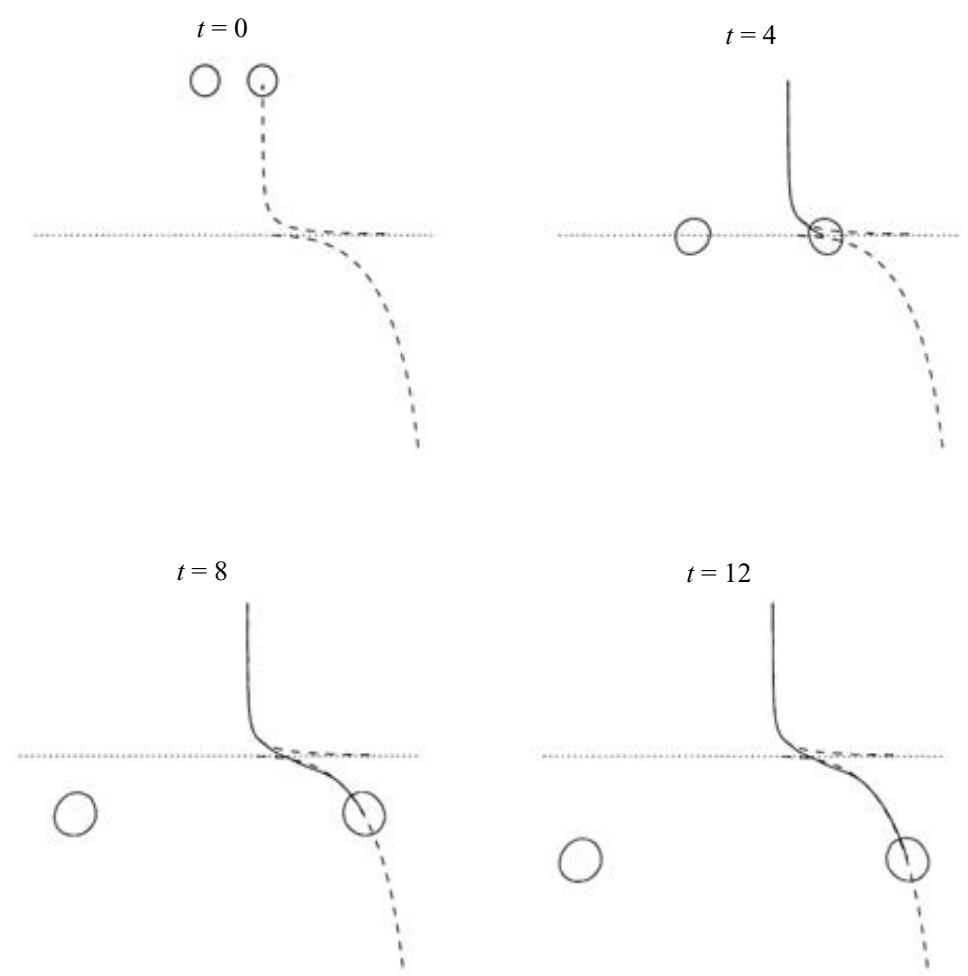

FiguRE 3. Evolution of a pair of initially circular vortex patches with radii 0.5 starting at $( \pm 1,5)$ for an upward step where the depth halves $\left(\gamma=H^{-} / H^{+}=0.5\right)$. The step lies along the dotted line $y=0$, the solid line is the trajectory of the centroid of the right-hand patch and the dashed line in $y>0$ the trajectory of the right-hand vortex of the singular vortex pair with the same circulation and initial centroid as the vortex patch. The dashed line in $y<0$ is the trajectory of this singular vortex determined uniquely by energy conservation for the vortex patch. Provided the patches remain approximately circular their centroids are constrained to closely follow the singular vortex paths.

monotonically to a finite maximum as the vortex crosses the step then decreases monotonically. For a given circulation smaller, more intense, vortices approach the step more closely before straddling the step than larger vortices, so their along-step velocity is greater, they deviate more from their initial path and they cross the step at a shallower angle. Only in the limit of zero-area singular vortices can vortices approach the step arbitrarily close without cutting the step. The along-step velocity of these singular vortices then increases arbitrarily, their angle of approach to the step approaches zero and they propagate almost parallel to the step.

Although the centroid constraint relaxes when a patch cuts a step, the energy $E$ of (2.4) is still conserved as finite-area vortices move along or across steps. In particular, irrespective of intermediate states, if the initial and final states of a vortex pair are circular (or approximately so), then their initial and final energies are the same and are given by (A 5) of the Appendix. This enables the asymptotic (for $-y \gg 1$ ) separation $\delta$ (say) of a vortex pair that has crossed a step to be predicted, given the initial size and position, as

$$
\log (\delta / b)=\gamma^{-1} \log \left\{(\Delta / a)\left[1+\left(2 \Delta / y_{i}\right)^{2}\right]^{\alpha / 2}\right\}+(1 / 4)\left(\gamma^{-1}-1\right),
$$

where $y_{i}$ is the initial distance of the pair from the step and $b$ is the final mean vortex 


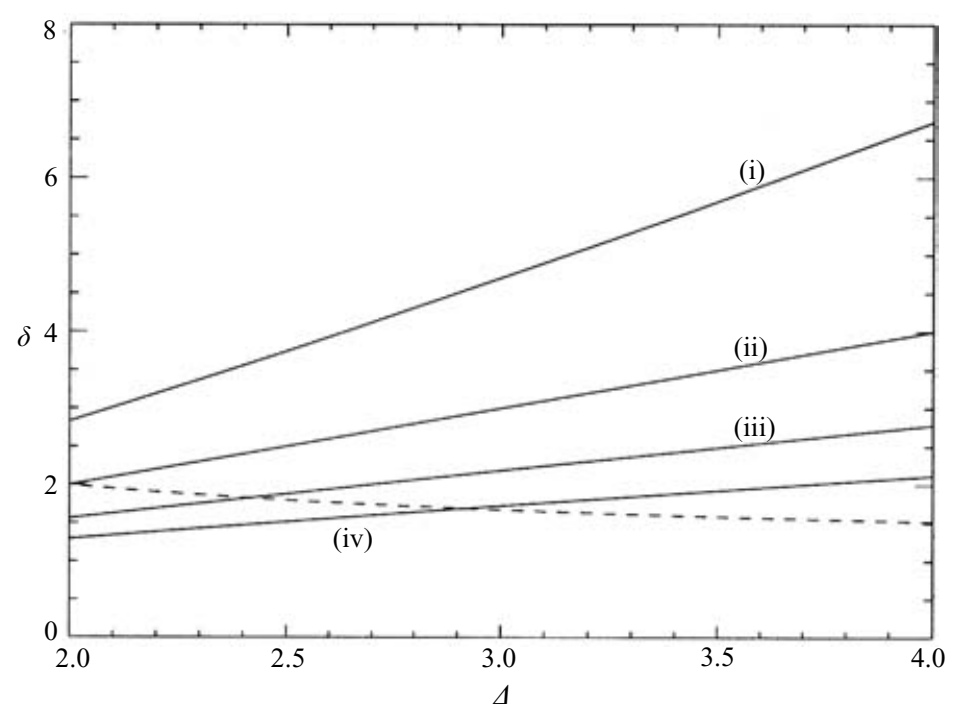

FIgURE 4. The centroid separation $\delta$ given by (4.1) for a vortex pair at large negative $y$ as a function of $\Delta$, their initial separation at large positive $y$. The vortices are assumed to be circular and the horizontal scale has been taken to be the initial vortex radius so $\Delta \geqslant 2$. (i) $\gamma=0.8$, an upward step; (ii) $\gamma=1$, no step; (iii) $\gamma=1.2$ and (iv) $\gamma=1.4$, downward steps. Below the dashed line vortices at large negative $y$ cannot remain circular without shedding vorticity.

radius, determined through conservation of volume as $b=a \gamma^{-1 / 2}$. Figure 4 show the variation of $\delta$ as a function of $\Delta$ for various depth ratios $\gamma$, where horizontal lengths have been scaled on the initial vortex radius and the vortices initially start at $y_{i} \gg 1$. The minimum value of $\Delta$ is 2 when the initial vortices touch. For $\gamma=1$, curve (ii), the step is absent, the vortex separation is constant and $\delta=\Delta$. For upward steps $(\gamma<1$, curve (i)) the vortices separate under the influence of their negative images, $\delta$ exceeds $\Delta$ and increases more rapidly. For downward steps $(\gamma>1$, curves (iii) and (iv)) the vortices move together due to their positive images, $\delta$ is less than $\Delta$ and increases more slowly. Below the dashed line, $\delta=2 \gamma^{-1 / 2}$, vortices must distort from circular to retain all their original vorticity or must shed vorticity on crossing the step. Combining (4.1) with (3.4) predicts the position of the centroid at all times on both sides of the step explicitly from the initial conditions, subject solely to the restriction that the vortex remains circular (or approximately so). For a head-on collision the patch separation always increases strictly monotonically for upward steps and decreases strictly monotonically for downward steps. This desingularization of singular vortex paths by associating the singular vortex with a circular patch of finite area in order to match paths on either side of the step is the discrete analogue of the numerical model in Zabusky \& McWilliams (1982) of continuously modulated vortices which are assigned areas in order to be affected by background vorticity or a sloping bottom.

Figure 3 shows the approach to an upward step $(\gamma=0.5)$ of a vortex pair of initially circular vortex patches of radius $a=1$. As the pair approaches the step the patches move apart under the influence of their images. The paths of the corresponding singular vortices (dashed line) and patch centroid (solid line) are virtually indistinguishable until the vortex mounts the step. Strikingly, however, the patches, 

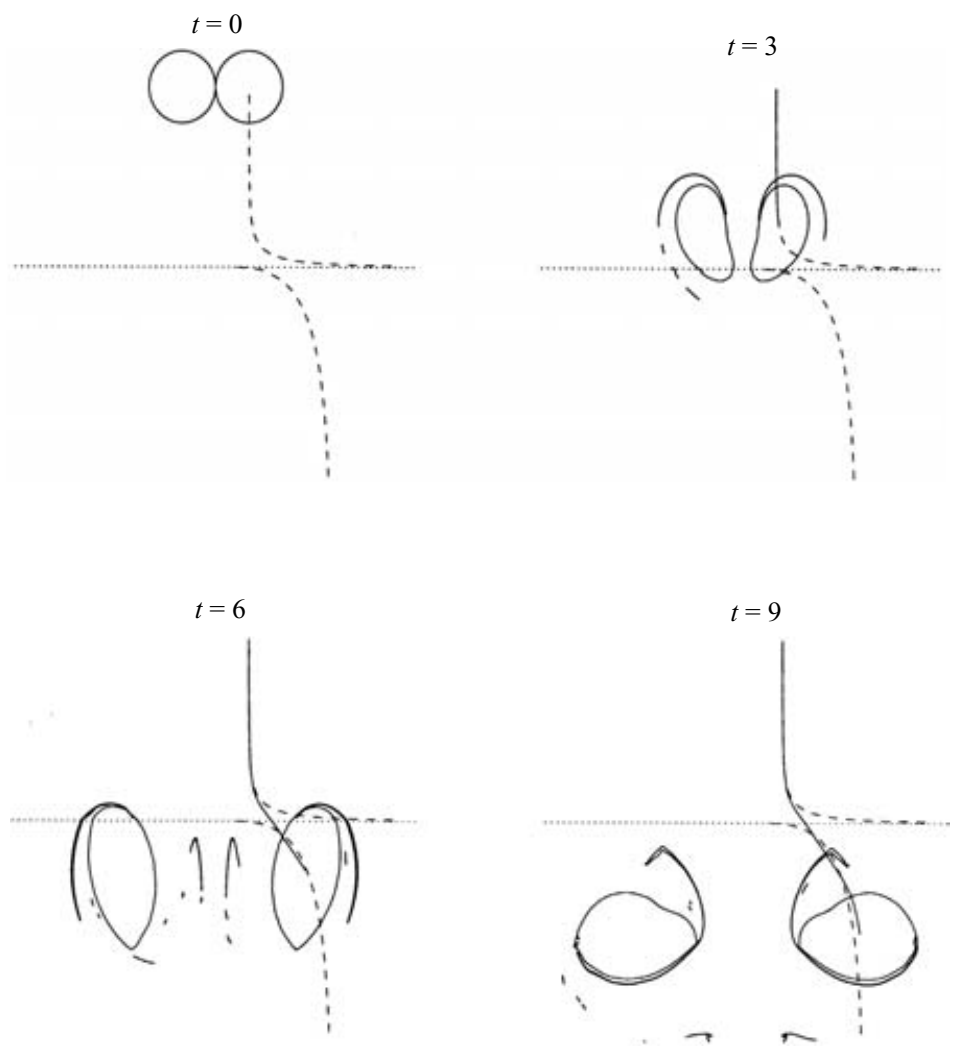

FIGURE 5. As in figure 3 except for initially touching circular patches starting at $( \pm 1,5)$ of maximal radius 1 . The patches travel faster and distort more. Their centroids still follow the paths of the equivalent singular vortices, although more closely before rather than after the now strong distortions and vortex shedding of crossing the step.

unlike the singular vortices, cross the step and continue to propagate in the same direction, albeit with a reduced speed owing to the increased separation of the two patches. The area of the patches also increases, to conserve mass, as they cross the step. The flow conserves energy even when the depth changes and so, provided the vortex returns to being approximately circular on the far-side of the step, the energy there is equal to the near-side energy and patch centroids are again constrained to follow the equivalent singular vortex paths for the new depth $\mathrm{H}^{+}$. These paths are given in $y<0$ in figure 3 and once the patch no longer cuts the step the centroid and singular vortex paths are almost indistinguishable.

Figure 5 shows the approach of an initially circular touching vortex patch pair (and so of maximal initial radii) towards the step for the same geometry as figure 3 . The propagation speed is larger for the larger (and so stronger) pair and since the patches are closer together they distort more, shedding vorticity even before cutting the step. The pair again crosses the step and the trajectories of the patch centroid and equivalent singular vortex pair compare well on both sides of the step, although less well after the vortex has been strongly distorted in passing over the step.

Figure 6 shows a vortex pair propagating from shallow water towards deep water. This time the step acts as an 'anti-wall' and under the influence of their 'anti-images' the patches approach each other as they near and then cross the step. The patch 

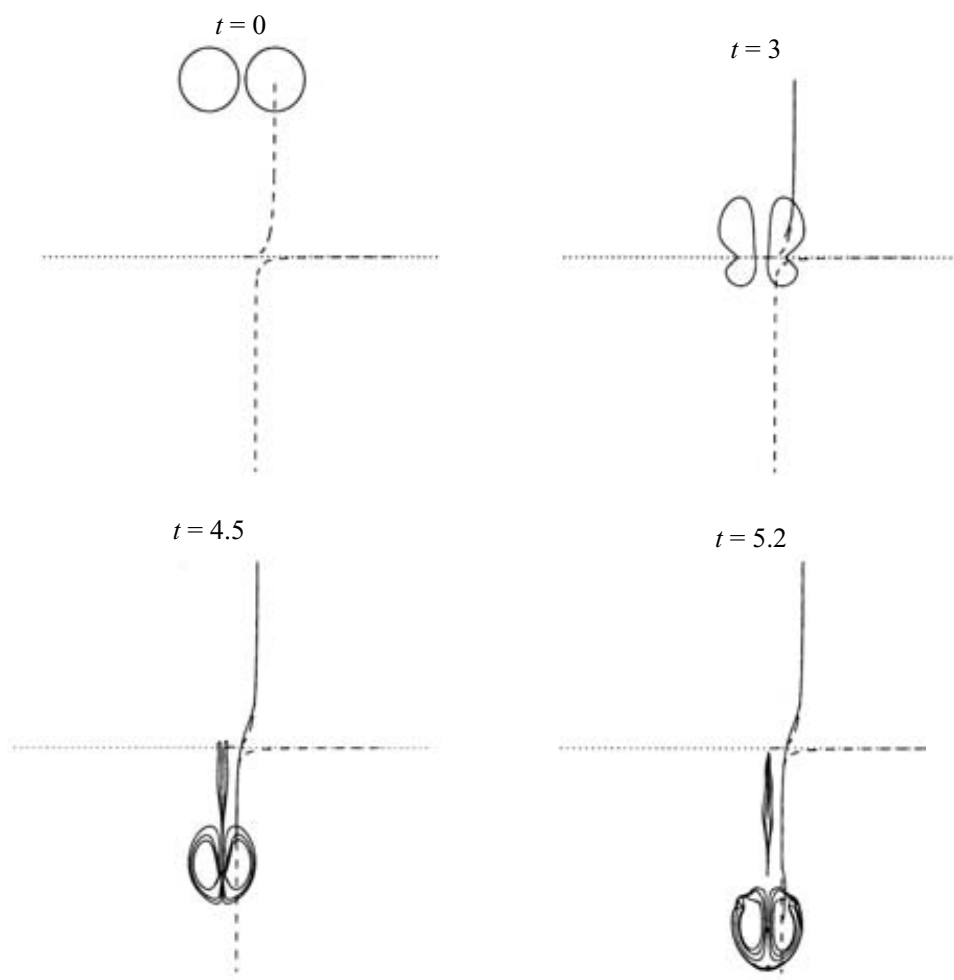

FIgURE 6. As in figures 3 and 5 but for patches of radius 0.9 and $\gamma=H^{-} / H^{+}=2$ so the depth doubles on crossing the step. Their centroids still follow the paths of the equivalent singular vortices, although more closely before rather than after the strong distortions and vortex shedding of crossing the step.

centroids follow the singular vortex paths closely both before and after the patches cross the step even though the patches shed a significant wake of vorticity on crossing the step. The pair continues to distort as it propagates away from the step and the final frame shows that this causes the centroid path to deviate slightly from the prediction.

In the examples above, the patch centroids closely follow the singular vortex predictions before collision with the step and any deviation occurs after the step. To show the effect of vortex size (or, equivalently, initial vortex separation) on this deviation figure 7 gives the centroid and singular vortex paths after a vortex has crossed an upward step (where the depth halves) for initially circular vortices with radii varying from $a=1$, the extreme touching case of figure 5 , to $a=0.4$. Although all centroids paths differ from the singular vortex paths while the patch lies above the step, the smaller patches soon readjust to closely follow the singular vortex paths. Even for the most extreme case the difference is only of order $5 \%$.

To show the effect of step height figure 8 gives the patch centroid and equivalent singular vortex paths for initially circular patches of radius $a=0.5$. The depth ratio $\gamma$ varies from $3 / 2$, where the depth decreases by a third, to 0.4 where the depth increases by a factor of $5 / 2$. A vortex of this size passes smoothly over the downward step and its path almost immediately becomes indistinguishable from the singular vortex path. The difference between this example and that of the upward step of figure 6 is that here there is sufficient room for the vortex pair to remain approximately circular after 


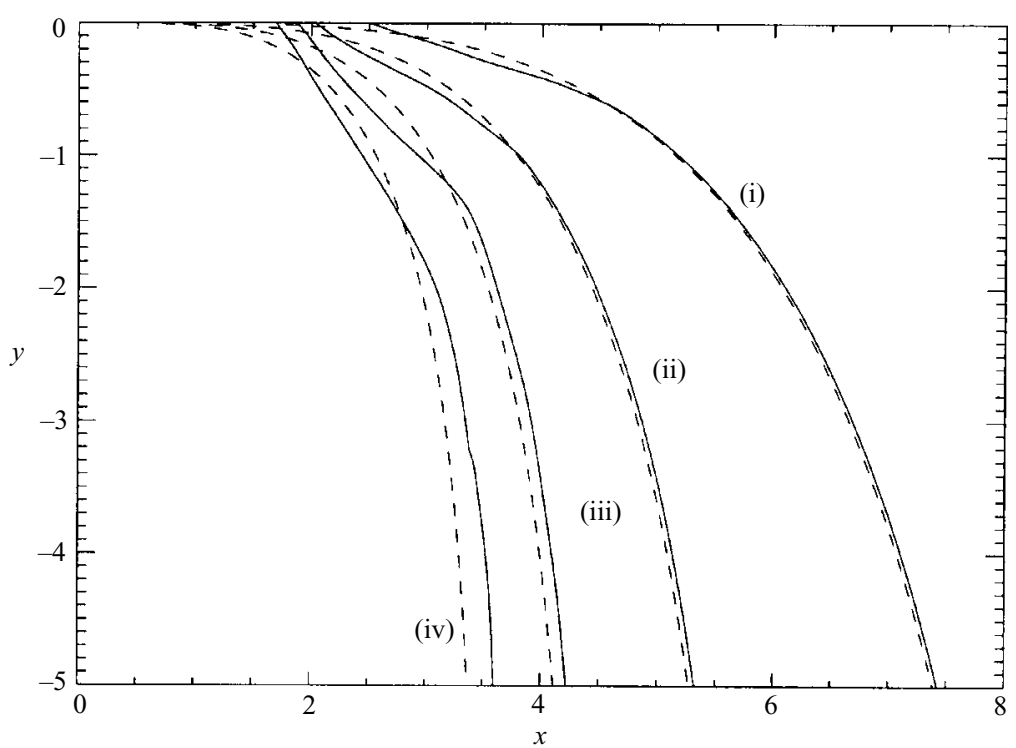

FiguRE 7. Trajectories (solid lines) of the centroid of a vortex patch making up one half a dipole pair after the patch has crossed the step. The dashed lines are the trajectories of singular vortices with equivalent energy and circulation. The depth halves at the step, $\gamma=H^{-} / H^{+}=0.5$, and the vortices are initially circular with radius $a$. (i) $a=0.4$. (ii) $a=0.6$. (iii) $a=0.8$. (i) $a=1.0$.

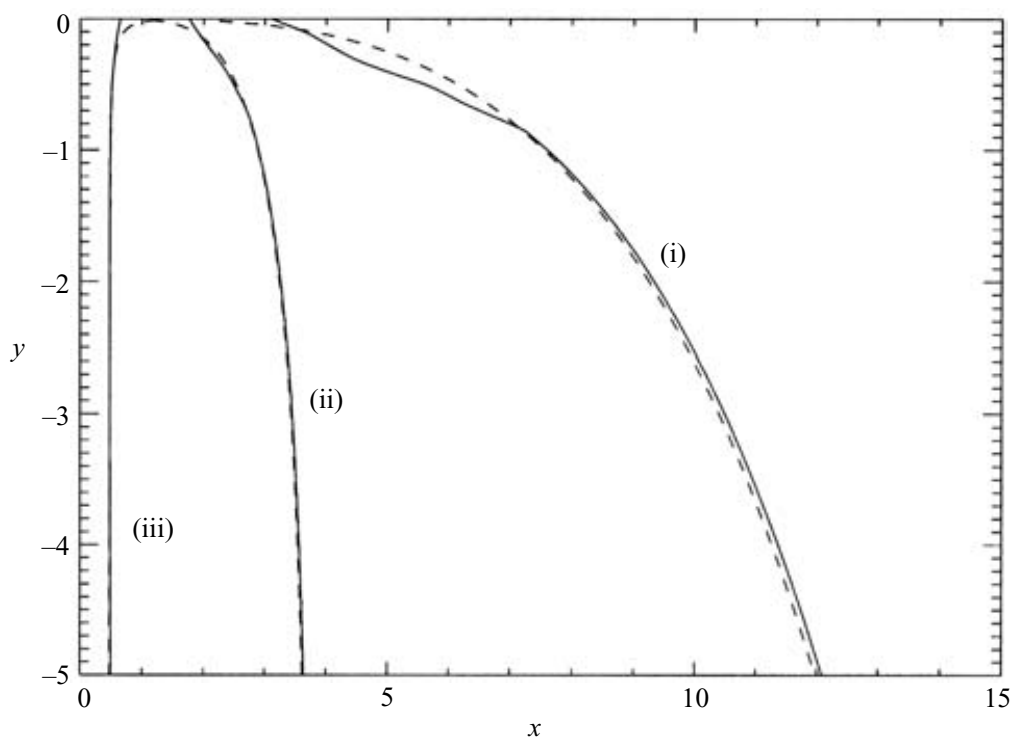

FiguRE 8. Trajectories (solid lines) of the centroid of a vortex patch making up one half a dipole pair for various values of depth ratio $\gamma=H^{-} / H^{+}$after the patch has crossed the step. The dashed lines are the trajectories of singular vortices with equivalent energy. In each case the patches are initially circular with radius $a=0.5$. (i) $\gamma=0.4$. (ii) $\gamma=0$.6. (i) $\gamma=1.5$.

crossing the step. In figure 6 the combined effects of the increase in horizontal area to conserve volume and the decrease in vortex separation on crossing the step mean that the vortex pair is forced to shed vorticity into a wake that is left behind. The 


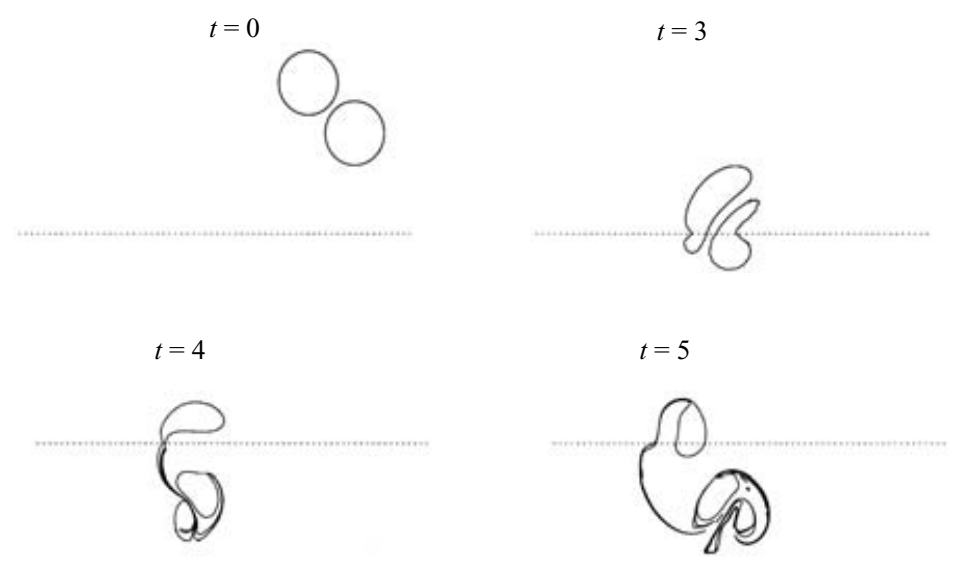

FIGURE 9. As in figure 6 but for a vortex patch dipole whose initial angle of incidence with the step is $\pi / 4$.

pair is also squashed becoming far from circular and closer to the shape of the almost touching vortices of Pierrehumbert (1980) and Saffman \& Tanveer (1982). The paths for patches passing over deepening steps coincide closely with singular vortex predictions although the paths for the largest depth increase take some time to settle to the singular vortex path due to the intensity of the image vorticity and thus to its strong effect near the step.

\section{Discussion}

The motion of surf vortices has been cast in a Hamiltonian framework for singular vortices above piecewise flat topography. This gives simple explicit formulae for single vortex paths and for groups of vortices if their initial arrangement has sufficient symmetry. Classical results relating the instantaneous vortex-induced flow fields, vortex velocities and Hamiltonians of conformally equivalent two-dimensional ideal fluid flows have been extended to shallow-water flow over finite-height topography. Simple examples of these results are given for a single finite step change in depth but the form of (3.1) shows that the extension to any arbitrary number of parallel finite steps is immediate, with the Green's function constructed simply by adding partial images. Singular vortex paths closely predict the motion of the centroid of finite-area vortex patches, with the main difference being that patches can cross the step. Even this crossing can, however, be modelled by associating a finite area with the singular vortex and considering energy conservation on crossing the step. It is important to note that no undetermined constants appear in the path predictions: the singular vortex paths on both sides of the step are uniquely determined by the initial position and size of the vortex patch.

Once the vortices have moved away from the step more accurate path models could be formed using higher-order-moment models, following Melander, Zabusky \& Styczek (1986) for constant-depth flow. However, once circulation, area, symmetry and energy are conserved, as here, it appears that there are no other conserved quantities to relate the moments after the collision with the step to those before. The symmetry of the head-on collisions studied here not only simplifies the singular vortex dynamics but also the vortex patch behaviour. Figure 9 shows a collision for the same vortex pair as figure 6 and the same downward step but for an oblique collision. The negative 
(left-hand side) patch tears on crossing the step. Part of the negative patch then pairs up with the positive patch which has crossed intact and this new pairing travels anti-clockwise along a curved path. The path curves as the absolute value of the circulation of the two patches differs, with the positive vortex dominating due to its larger size. By the final frame the pair is moving back towards the step. The remainder of the negative patch now begins to cross the step to temporarily form a tri-polar structure. Conservation of momentum in the $x$-direction means that it is impossible for parts of the original vortices to be left behind at the step while other parts propagate away unless the propagating parts have zero total circulation. Integrations for other angles of incidence and depth ratios show the same general behaviour. It is difficult to see how a moment model could capture this.

Self-advecting dipole structures have been suggested as models for surf-zone rip currents - see, for example, Arthur (1962) and Peregrine (1998). Such currents play an important role in the transport of fluid and sediment in the surf zone and it is of interest to understand their behaviour, especially in regions of rapidly varying topography such as occurs on barred beaches. Barred beaches may be crudely modelled by step topography of the type considered here. The results show that rip currents may intensify as they propagate into deeper regions. As noted by Richardson (2000) for smooth topography, the intensification is caused by the coming together of the two halves of the dipole to give a larger propagation speed. Dipoles entering shallower water separate and slow down. In both geometries the propagation speeds and trajectories of the vortices comprising the dipoles are well, modelled by singular vortices of equivalent energy.

We are indebted to Professor Dritschel for providing us with a copy of his 1988 code that formed the basis for the code developed here. E. R. J. is also indebted to the School of Mathematical Sciences at Monash University for their hospitality while some of this work was carried out and to the UK Natural Environment Research Council for their support through Grant NER/A/S/2000/01323.

\section{Appendix. Energy conservation for a vortex pair approaching a step}

Consider a pair of circular vortices of constant potential vorticity $\pm Q$, radius $a$ and separation of centres $\Delta$. Then the streamfunction due to the single vortex of strength $Q$ can be written in terms of the distance $r$ from its centre as

$$
\psi= \begin{cases}-(V Q H / 2 \pi)\left[\frac{1}{2}(r / a)^{2}-\frac{1}{2}\right]+C, & r<a \\ -(V Q H / 2 \pi) \log (r / a)+C, & r>a,\end{cases}
$$

where $V$ is the constant volume of the vortex and $C$ is an arbitrary constant.

The energy is the sum of the two equal self-interaction energies of the vortices plus the two equal cross-vortex interaction energies. The cross-vortex energy is simply

$$
\frac{1}{2}\left\{-Q H \pi a^{2}[(-V Q H / 2 \pi) \log (\Delta / a)+C]\right\}=\frac{1}{2} Q V[(V Q H / 2 \pi) \log (\Delta / a)-C] .
$$

The self-interaction energy is

$$
\frac{1}{2} Q H\left[(V Q H / 2 \pi)\left\{-2 \pi \int_{0}^{a}\left[\frac{1}{2}(r / a)^{2}-\frac{1}{2}\right] r \mathrm{~d} r+\pi a^{2} C\right]\right\}=\frac{1}{2}\left(Q^{2} V^{2} H / 2 \pi\right)(1 / 4+C) .
$$


Adding these terms for both vortices gives

$$
E=\left(Q^{2} V^{2} H / 2 \pi\right)[\log (\Delta / a)+1 / 4],
$$

which is independent of $C$ as expected.

For a vortex pair approaching a step change in depth (A 4) gives the energy of the pair away from the step. Close to the step the images in the step contribute to the energy. The terms have precisely the form (A 2) with, however, strengths $\pm \alpha$ and centre separation distances $y_{S}$ (say) and $r_{S}=\left(\Delta^{2}+y_{S}^{2}\right)^{1 / 2}$. Thus the expression for the energy becomes

$$
E_{C}=\left(Q^{2} V^{2} H / 2 \pi\right)\left\{\log \left[(\Delta / a)\left|r_{S} / y_{S}\right|^{\alpha}\right]+1 / 4\right\} .
$$

This is of exactly the form of the Hamiltonian (3.3) for singular vortices, since the total potential vorticity in any horizontal plane is here $\pi a^{2} Q$, corresponding to $Q$ in (3.3), and so $Q^{2} V^{2}$ in (A 5) becomes $Q^{2} H^{2}$ in (3.3).

\section{REFERENCES}

ArthuR, R. S. 1962 A note on the dynamics of rip currents. J. Geophys. Res. 67, 2777-2779.

Bidlot, J. R. \& Stern, M. E. 1994 Maintenance of continental boundary-layer shear through counter-gradient vorticity flux in a barotropic model. J. Fluid Mech. 271, 55-85.

BüHLER, O. \& JACOBSON, T. E. 2001 Wave-driven currents and vortex dynamics on barred beaches. J. Fluid Mech. 449, 313-339.

Chen, Q., Dalrymple, R. A., Kirby, J. T., Kennedy, A. B. \& Haller, M. C. 1999 Boussinesq modeling of a rip current system. J. Geophys. Res. 104, 20617-20637.

DritsChel, D. G. 1988 Contour surgery: a topological reconnection scheme for extended contour integrations using contour dynamics. J. Comput. Phys. 77, 240-266.

Gorshkov, K. A., Ostrovsky, L. A. \& Soustova, I. A. 2000 Perturbation theory for Rankine vortices. J. Fluid Mech. 404, 1-25.

Hinds, A. K., Johnson, E. R. \& McDonald, N. R. 2004 Beach vortex V-states. In preparation.

Johnson, E. R. 1978 Trapped vortices in rotating flow. J. Fluid Mech. 86, 209-224.

Johnson, E. R. 1985 Topographic waves and the evolution of coastal currents. J. Fluid Mech. 160, 499-509.

JoHnson, E. R. 1987 A conformal-mapping technique for topographic-wave problems - semi-infinite channels and elongated basins. J. Fluid Mech. 177, 395-405.

Johnson, E. R. 1990 The low-frequency scattering of Kelvin-waves by stepped topography. J. Fluid Mech. 215, 23-44.

Johnson, E. R. 1993 Low-frequency scattering of Kelvin waves by continuous topography. J. Fluid Mech. 248, 173-201.

Johnson, E. R. \& McDonald, N. R. 2004a The motion of a vortex near a gap in a wall. Phys. Fluids 16, 462-469.

Johnson, E. R. \& McDonald, N. R. $2004 b$ The motion of a vortex near two circular cylinders. Proc. R. Soc. Lond. A 460, 939-954.

Lamb, H. 1932 Hydrodynamics, 6th edn. Cambridge University Press.

Melander, M., Zabusky, N. \& Styczek, A. 1986 A moment model for vortex interactions of the two-dimensional Euler equations, Part i., Computational validation of a Hamiltonian elliptical representation. J. Fluid Mech. 167, 95-115.

ÖzKan-Haller, H. T. \& Kirby, J. T. 1999 Nonlinear evolution of shear instabilities of the longshore current: A comparison of observations and computations. J. Geophys. Res. 104, 25953-25984.

Pedlosky, J. 1986 Geophysical Fluid Dynamics. Springer.

Peregrine, D. H. 1998 Surf zone currents. Theoret. Comput. Fluid Dyn. 10, 295-309.

Peregrine, D. H. 1999 Large-scale vorticity generation by breakers in shallow and deep water. Eur. J. Mech. B 18, 403-408.

Pierrehumbert, R. T. 1980 A family of steady, translating vortex pairs with distributed vorticity. J. Fluid Mech. 99, 129-144. 
RichARDSON, G. 2000 Vortex motion in shallow water with varying bottom topography and zero Froude number. J. Fluid Mech. 411, 351-374.

Routh, E. J. 1881 Some applications of conjugate functions. Proc. Lond. Math. Soc. 12, 73-89.

Saffman, P. G. 1992 Vortex Dynamics. Cambridge University Press.

Saffman, P. G. \& TanveEr, S. 1982 The touching pair of equal and opposite uniform vortices. Phys. Fluids 25, 1929-1930.

Slinn, D. N., Allen, J. S., Newberger, P. A. \& Holman, R. A. 1998 Nonlinear shear instabilities of alongshore currents over barred beaches. J. Geophys. Res. 103, 18357.

Smith, J. A. \& LARGier, J. L. 1995 Observations of nearshore circulation: Rip currents. J. Geophys. Res. 100, 10967-10975.

TAIT, P. 1867 Translation of 'On integrals of the hydrodynamical equations which express vortexmotion' by H. Helmholtz. Phil. Mag. 33, 485-512.

Thorpe, S. A. \& Centurioni, L. R. 2000 On the use of the method of images to investigate nearshore dynamical processes. J. Mar. Res. 58, 779-788.

Zabusky, N. J. \& MCWilliams, J. C. 1982 A modulated point-vortex model for geostrophic, beta-plane dynamics. Phys. Fluids 25, 2175-2182. 